\title{
Announcing the return of Protocol Review
}

\author{
The column will soon continue on with two new coordinators at the helm.
}

ast fall, the lab animal community
lost an invaluable member with the
passing of Jerald Silverman, IACUC extraordinaire and the decades-long coordinator of Lab Animal's Protocol Review column. We've noticed the absence in our pages and so have you, and while Jerry leaves a remarkable legacy, we are very pleased to announce that the column he dedicated all those years to will continue on. Here, we'd like to introduce you to the new coordinators who will be leading the coming iteration of Protocol Review: Lauren Danridge and Bill Greer.

Lauren Danridge serves as the Associate Director of the Animal Care \& Use Office at the University of Michigan. Lauren joined the Animal Care \& Use Office in September 2017 as the Assistant Director of the new Quality Assurance Program; in January 2018, she was named Associate Director. Lauren came to the University of Michigan from Rutgers University (formerly UMDNJ), where she served as both the Manager and Assistant Manager for Institutional Animal Care \& Use Committee Administration.

In her role as Associate Director at the University of Michigan, she supports the Animal Care \& Use Program by assisting in the leadership and management of oversight programs for the protection of animals involved in research at the University. Current programmatic goals are to reduce regulatory burden, continue engaging the research community in programmatic improvements, and streamlining business processes to increase efficiency and effectiveness. Lauren has over 20 years of experience in laboratory animal research and compliance, including hands-on animal research and IACUC Administration. Lauren also has served as a research administrator in basic science departments, responsible for managing a variety of administrative processes, including preand post-award grant activities, contracts, manuscripts, presentations, and protocols. As an active contributor to the national animal care and use community and higher education institutions, Lauren regularly presents on research administration best practices for IACUC, occupational health and safety, and biosafety.

Bill Greer currently serves as the Assistant Vice President for Research at the University of Michigan. His previous roles included Associate Director for Research Compliance at the Pennsylvania State University, and research technician, production manager and Safety Officer at Intervet, State College, PA (formally Tri Bio Laboratories). In 2005, he organized and held the first IACUC Administrators' Best Practices Meeting, which establish a venue for administrators to meet informally and discuss programmatic concerns. $\mathrm{He}$ continues to facilitate at least three annual Best Practice meetings. In 2007, he initiated the process of establishing the IACUC Administrators Association (IAA), which is a professional organization of IACUC Administrators. In 2010, he chaired the founding committee for the non-profit education based IAA organization. $\mathrm{He}$ now serves as the president and chair of the IAA board of directors. Since 2007, Bill has served as ad hoc specialist to the AAALAC International Council where he participates in institutional program reviews, assessments and status determinations. He served as a member of the Council of Certified Professional IACUC Administrators (CCPIA). He continues to facilitate training activities at multiple venues across the IACUC and Biosafety Compliance Administrators communities.

We've asked them to share a few thoughts about why they decided to step up to the Protocol Review plate:

"Due to the nature of the regulations governing animal care and use (i.e., the inherent flexibilities and prolific "grey areas"), the IACUC Community relies heavily on a variety of resources for information and guidance; Jerry's Protocol Review Column was a primary resource. Anyone who had the privilege of meeting Jerry would know that he truly cared about the IACUC community and its mission.

Everyone in the IACUC Community has benefited, in some fashion or another, from each other's input, advice, and efforts - from seeking advice to sharing documents. This collegiality relies on folks stepping up and putting in the effort to keep the community engaged and interactive. When it seemed that the community might lose the column that Jerry developed and nurtured over the years, we knew we needed to do our part and carry the Protocol Review Column-baton forward."

These are no small shoes to fill, but we are confident Lauren and Bill are more than up to the challenge. In the coming months, they will be working on putting their own spin on the column but just as before, its success very much relies on you. If you'd like to contribute to an upcoming column, our new coordinators are prepping their virtual rolodex of volunteers - from the United States and from among the international animal research community as well as brainstorming potential scenarios to pose to readers.

We encourage you to share your interest and/or any problems you and your institution are pondering with Lauren (danridlm@umich.edu) and Bill (wggreer@umich.edu). Please reach out, and keep your eyes peeled for the return of Protocol Review.

Published online: 28 July 2021

https://doi.org/10.1038/s41684-021-00824-w 